\title{
Cirugía de revisión femoral con vástagos monobloque de recubrimiento completo. Resultados a largo plazo de 78 casos
}

\author{
Femoral revision surgery with fully coated stems. \\ Long-term results of 78 cases \\ Diranzo-García J,* Hernández-Ferrando L, „Estrems-Díaz V, \\ Castillo-Ruipérez L,* Zarzuela-Sánchez VM,* Bru-Pomer A ${ }^{\S}$
}

Hospital General Universitario de Valencia, España.

RESUMEN. Introducción: La pérdida de stock óseo y la alteración anatómica del fémur proximal hacen de la cirugía de revisión femoral un procedimiento complejo en la que la elección del implante será fundamental. Nuestro objetivo es valorar los resultados clínicos y radiológicos de los vástagos monobloque de recubrimiento completo. Material y métodos: Estudio retrospectivo de 78 casos consecutivos de cirugía de revisión femoral intervenidos por nuestra unidad. Resultados: El seguimiento medio fue de 122 meses. La puntuación media en la escala de Harris Hip Score fue de $81.2 \pm 11.4$ puntos. Una edad menor de 65 años, un defecto femoral menor según la clasificación de Paprosky (I, II y IIIA), la existencia de una única cirugía previa y la no aparición de complicaciones intraoperatorias o postoperatorias se asoció con mejores resultados clínicos y funcionales ( $\mathrm{p}$ $<0.05$ ). La supervivencia acumulada fue de $96.7 \%$ a los 10 años si consideramos como fracaso la retirada del vástago por aflojamiento aséptico y de $92 \%$ si consideramos como fracaso la retirada por cualquier causa. Conclusión: Pese a la tendencia actual al uso de la modularidad en cirugía de revisión femoral y dados los resultados presentados, con-
ABSTRACT. Introduction: Loss of bone stock and anatomical alteration of the proximal femur make femoral revision surgery a complex procedure in which the choice of implant will be critical. Our goal is to assess the clinical and radiological results of complete coating Monoblock stems. Material and methods: Retrospective study of 78 consecutive cases of femoral review surgery intervened by our unit. Results: The average follow-up was 122 months. The average score on the Harris Hip Score was $81.2 \pm 11.4$ points. An age of less than 65 years, a minor femoral defect according to Paprosky classification (I, II and IIIA), the existence of a single previous surgery and the non-occurrence of intra or postoperative complications, was associated with better clinical and functional outcomes $(\mathrm{p}<0.05)$.Cumulative survival was $96.7 \%$ at age 10 if we consider as failure the removal of the stem by aseptic loosening and 92\% if we consider withdrawal for any cause to fail. Conclusion: Despite the current tendency to use modularity in femoral review surgery and given the results presented, we consider that complete coating monoblock stems provide a firm and stable fixation. However, the

\section{Nivel de evidencia: IV}

* Unidad de Cadera y Pelvis.

‡ Jefe Clínico Unidad de Cadera y Pelvis.

$\S$ Jefe de Servicio.

Hospital General Universitario de Valencia, España.

Correspondencia:

Dr. José Diranzo-García

Hospital General Universitario de Valencia. Avenida de las Tres Cruces 2, 46014, Valencia, España.

E-mail: pdiranzo@gmail.com

Recibido: 07-10-2020. Aceptado: 21-02-2021.

Citar como: Diranzo-García J, Hernández-Ferrando L, Estrems-Díaz V, Castillo-Ruipérez L, Zarzuela-Sánchez VM, Bru-Pomer A. Cirugía de revisión femoral con vástagos monobloque de recubrimiento completo. Resultados a largo plazo de 78 casos. Acta Ortop Mex. 2021; 35(1): 33-39. https://dx.doi.org/10.35366/100928 
sideramos que los vástagos monobloque de recubrimiento completo proporcionan una fijación firme y estable. Sin embargo, los peores resultados obtenidos en pacientes con defectos óseos mayores han provocado que se valore otro tipo de opciones.

Palabras clave: Cadera, artroplastía, revisión, cirugía, éxito.

\section{Introducción}

Se prevé que el número de cirugías de revisión tras artroplastía de cadera se incremente $137 \%$ entre 2005 y 2030, ${ }^{1}$ debido principalmente al envejecimiento poblacional y a la utilización de la artroplastía en pacientes de menor edad y con mayor demanda funcional. ${ }^{2}$ El aflojamiento aséptico del vástago supone la principal causa de cirugía de revisión femoral, seguida de la infección, las fracturas periprotésicas y la luxación recidivante. ${ }^{3,4}$

Tradicionalmente, los vástagos monobloque de recubrimiento completo han constituido una de las principales alternativas de rescate habiendo sido publicadas tasas de supervivencia a medio/largo plazo de hasta $97.5 \%$ tras la revisión del componente femoral con este tipo de implantes. ${ }^{5}$ Su estructura cilíndrica y sin reducción del diámetro distal favorece la fijación primaria en hueso diafisario sano, baipaseando el defecto proximal. La aparición de stress-shielding y la elevada incidencia de dolor de muslo (hasta 10\%) asociada a los primeros diseños, ${ }^{6}$ rectos y de una rigidez superior al hueso por su construcción en cromo-cobalto ha conllevado una evolución hacia los modelos utilizados en la actualidad fabricados en titanio y con un radio de curvatura similar al del fémur del paciente.

El propósito del presente estudio fue valorar los resultados clínicos y radiográficos, así como la incidencia de complicaciones en una serie de 78 casos en 75 pacientes consecutivos intervenidos con vástagos monobloque de worst results in patients with major defects have led to other options being considered.

Keywords: Hip, replacement, revision, surgery, success.

recubrimiento completo, con un seguimiento mínimo de cinco años.

\section{Material y métodos}

Entre Marzo de 2005 y Noviembre de 2017 se realizaron 161 cirugías de revisión femoral en 151. De los 151 pacientes, 16 fallecieron por causas no relacionadas con la cirugía y 60 no alcanzaron un seguimiento mínimo de cinco años, restando un total de 75 pacientes y 78 cirugías de revisión para el presente estudio. Todos los pacientes firmaron el correspondiente consentimiento informado.

Análisis estadístico: el tratamiento de los datos se ha llevado a cabo mediante los programas estadísticos SPSS 22 y XLSTAT para MAC OS. El análisis descriptivo de las variables categóricas se expresa como frecuencia absoluta y relativa; sin embargo, las variables cuantitativas se describen con media y la desviación estándar (DE). Se comprobó la normalidad de las variables cuantitativas mediante la prueba de Kolmogorov-Smirnov.

Para el análisis de las variables cuantitativas se aplica el test paramétrico $t$ de Student-Fisher. La tasa de supervivencia acumulada del implante se evaluó mediante el método de Kaplan-Meier, considerando como eventos finales: la retirada del vástago por aflojamiento aséptico, la retirada del vástago por cualquier causa y la revisión del vástago por cualquier razón o migración del mismo superior a $5 \mathrm{~mm}$. En todos los análisis estadísticos el nivel de significación se estableció en 5\%.
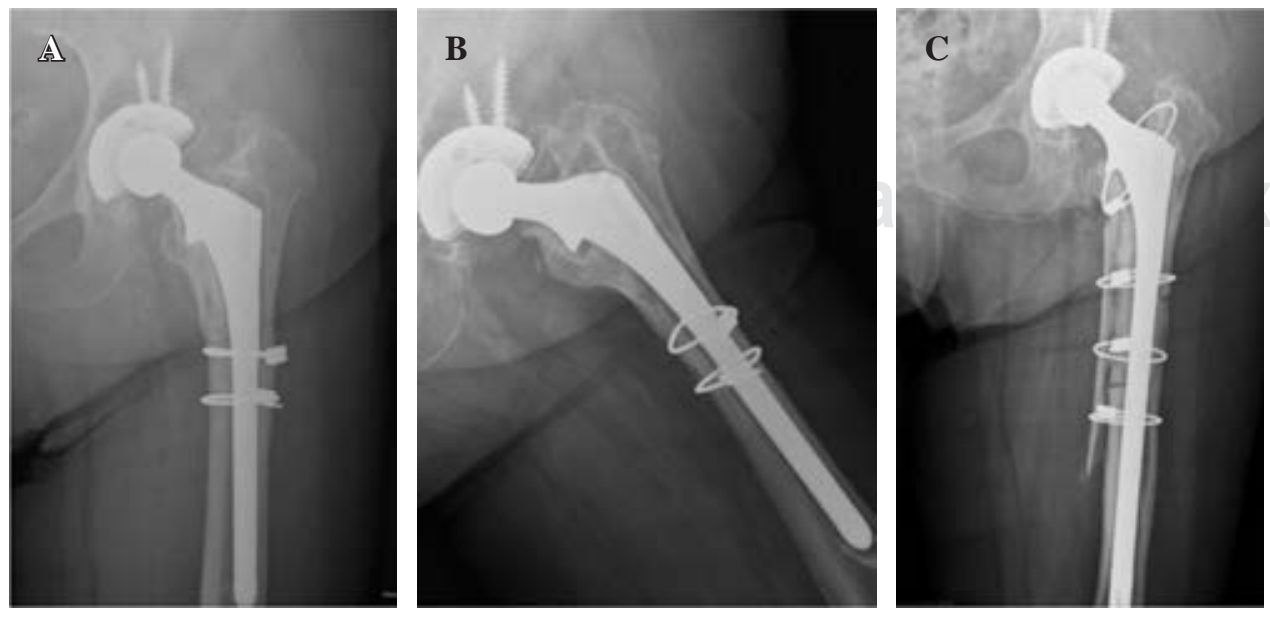

Figura 1:

Aflojamiento aséptico A) y B) recambiado por vástago con un diámetro y longitud mayor. Aloinjerto estructural con cerclajes para reparar de la osteotomía trocantérica C). 


\begin{tabular}{|c|c|c|c|c|c|}
\hline Tipo defecto & I & II & IIIA & IIIB & Total \\
\hline \multicolumn{6}{|c|}{ Diámetro (mm) } \\
\hline 11 & 2 & - & 3 & - & 5 \\
\hline 12 & 2 & 9 & 1 & 2 & 14 \\
\hline 13 & 3 & 11 & 7 & 5 & 26 \\
\hline 14 & - & 3 & 6 & 3 & 12 \\
\hline 15 & 2 & 1 & 8 & 4 & 15 \\
\hline 16 & - & - & 3 & 1 & 4 \\
\hline 17 & - & - & - & 2 & 2 \\
\hline Total & 9 & 24 & 28 & 17 & 78 \\
\hline \multicolumn{6}{|c|}{ Longitud (mm) } \\
\hline $155^{\circ}$ & 2 & 4 & 4 & - & 10 \\
\hline 205 & 4 & 15 & 13 & 8 & 40 \\
\hline 255 & 3 & 5 & 11 & 9 & 28 \\
\hline Total & 9 & 24 & 28 & 17 & 78 \\
\hline
\end{tabular}

Técnica quirúrgica: la planificación preoperatoria se realizó mediante el uso de plantillas con el objetivo de determinar la longitud y diámetro del vástago necesarios para obtener un contacto cortical de $4 \mathrm{~cm}$ y una ocupación mínima de 90\% del canal femoral. En todas las revisiones se realizó un fresado del canal femoral 0.5 mm inferior al diámetro del implante definitivo.

En todas se usó el abordaje posterolateral a la cadera. Realizamos una osteotomía trocantérica ampliada (OTA) en 19 casos (24.4\%). La fijación de la osteotomía se realizó en todos los casos mediante cerclajes asociando en tres pacientes osteosíntesis con placa trocantérica y en 11 casos aloinjerto estructural criopreservado (Figura 1). En 42 casos se llevó a cabo de forma concomitante una revisión del componente acetabular, implantándose en 17 casos cotilos de doble movilidad, en 24 cotilos hemiesféricos monopolares y en un caso un cotilo retentivo.

Evaluación postoperatoria: todos los pacientes fueron evaluados a las tres, seis semanas, tres, seis meses y posteriormente cada año para examen clínico y seguimiento radiográfico. En cada visita se incluyó la escala de valoración funcional de Harris, ${ }^{7}$ el grado de satisfacción del paciente, la presencia de dolor en actividades cotidianas, la capacidad para la deambulación y radiografías simples (AP de pelvis en bipedestación y AP y lateral de cadera y fémur intervenidos). Valoramos la integración del componente femoral siguiendo los criterios radiológicos de Engh $^{8}$ y consideramos como hundimiento del vástago una migración distal del mismo superior a $5 \mathrm{~mm}$. Asimismo, determinamos la presencia de stress-shielding proximal basándonos en los criterios de Engh y Bobyn ${ }^{9}$ y el desarrollo de osificaciones heterotópicas según Brooker. ${ }^{10}$ Registramos la aparición de complicaciones tales como fractura femoral, drenaje persistente de la herida quirúrgica, infección y luxación. Por último, evaluamos la necesidad de reintervención por cualquier causa y de re-revisión del componente femoral.

\section{Resultados}

El tiempo medio de seguimiento de los 78 casos (75 pacientes) fue de 122 meses (rango, 60-186 meses). De los 75 pacientes, 29 (39\%) eran varones y 46 (61\%) mujeres. La edad media en el momento de la cirugía fue de 67.6 años (rango, 33-88 años). El índice de masa corporal (IMC) medio de la muestra fue de $29.1 \mathrm{~kg} / \mathrm{m}^{2}$ (rango, $14-38 \mathrm{~kg} / \mathrm{m}^{2}$ ). Del total de pacientes, 45 (60\%) presentaban enfermedades sistémicas asociadas, destacando 21 pacientes (28\%) con enfermedades cardiopulmonares y 15 pacientes (20\%) con obesidad severa (IMC 35-39.9).

La etiología para la cirugía de revisión femoral fue el aflojamiento aséptico en 45 casos (60\%), infección protésica en 14 casos (17.9\%), fracaso de osteosíntesis tras fractura de fémur proximal en 10 casos (12.8\%), fractura periprotésica de fémur en ocho casos (10.3\%) y rotura del vástago en un caso (1.3\%). El número medio de cirugías protésicas previas en la cadera intervenida fue de 1.6 cirugías: 47 casos (60.3\%) habían sido intervenidos una única vez, mientras que 31 (41.3\%) habían sufrido de dos hasta un máximo de cinco cirugías previamente. La fijación del vástago extraído era en 43 casos (63.2\%) press-fit y en 25 casos (36.8\%) cementada. Excluimos los 10 casos intervenidos tras un fracaso de osteosíntesis. El defecto femoral preoperatorio fue clasificado en nueve casos (11.5\%) como Paprosky tipo I, en $24(30.8 \%)$ como tipo II, en 28 casos (35.9\%) tipo IIIA y en 17 (21.8\%) como tipo IIIB. Las fracturas periprotésicas fueron clasificadas según los criterios de Vancouver, ${ }^{11}$ siendo dos de ellas tipo B1, cinco tipo B2 y una tipo B3.

El implante femoral utilizado en todos los casos fue el vástago de revisión Restoration $\mathrm{HA}^{\circledR}$ con recubrimiento completo de hidroxiapatita (Stryker, Mahwah, NJ). La distribución del diámetro de los vástagos utilizados se muestra en la Tabla 1. El tiempo quirúrgico medio fue de $107 \mathrm{mi}-$ nutos (rango, 85-140 minutos) en las cirugías de revisión femoral aislada y de 138 minutos (rango, 110-180 minutos) en revisión acetabular y femoral. Del total de pacientes, 54 (69.2\%) precisaron ser transfundidos con una media de 2.6 unidades de concentrados de hematíes durante la intervención o en el postoperatorio inmediato.

Resultados clínicos: la puntuación en la escala de Harris mejoró de $39.4 \pm 12.7$ puntos sobre 100 preoperatorios a 81.2 puntos (DE 11.4 puntos) en último control ( $\mathrm{p}=0.001$ ). No fueron objetivadas diferencias significativas en la puntuación en la escala de Harris postoperatoria en cuanto al sexo del paciente o índice de masa corporal. Sin embargo, comprobamos que los pacientes menores de 65 años son los que presentaban defectos femorales de menor severidad (Paprosky tipos I, II y IIIA); aquéllos en los que se trataba de la primera cirugía de revisión y los que no sufrieron complicaciones postoperatorias obtuvieron resultados clínicos significativamente mejores $(\mathrm{p}<0.05)$ (Tabla 2).

Del total de pacientes, $81.3 \%$ se mostraron satisfechos con la cirugía y 76\% manifestaron no tener dolor en sus actividades cotidianas. Del total de 75 pacientes, 36 deambu- 
laban sin asistencias mecánicas, 17 con ayuda de un bastón/ muleta, 18 con andador o dos muletas y cuatro eran incapaces de deambular.

Resultados radiológicos: tres de los 78 vástagos (3.8\%) desarrollaron un aflojamiento aséptico con un hundimiento superior a $5 \mathrm{~mm}$ en las radiografías estándar, obteniendo una correcta osteointegración en los 75 restantes (96.2\%). Dos de los tres casos de aflojamiento se dieron en fémures con defecto Paprosky Tipo IIIB y uno en IIIA. Dos de ellos fueron reintervenidos mediante una revisión femoral con un vástago de mayor diámetro y el tercero rechazó una nueva cirugía.

Del total de pacientes, 28 casos (35.9\%) desarrollaron una osteopenia del fémur proximal por stress-shielding sin que ello tuviese una traducción clínica significativa (Tabla 2). Objetivamos la aparición de osificaciones heterotópicas en 15 casos (19.2\%): ocho tipo I de Brooker, dos tipo II, tres tipo III y dos tipo IV. Ningún paciente precisó ser reintervenido por este motivo.

Complicaciones: 19 pacientes (25.3\%) sufrieron un total de 20 complicaciones. Entre las complicaciones intraoperatorias destacamos nueve fracturas: seis fracturas no desplazadas del calcar femoral tratadas mediante cerclajes, dos perforaciones de la cortical anterior que no requirieron ningún procedimiento añadido y una fractura tipo C de Vancouver tratada mediante placa bloqueada.

En cuanto a las complicaciones postoperatorias, incluimos una fractura periprotésica B1 de Vancouver tratada con placa atornillada y cerclajes; cinco casos (6.4\%) de luxación (tres tratados mediante reducción cerrada y dos mediante re-

\begin{tabular}{|c|c|c|}
\hline \multicolumn{3}{|c|}{$\begin{array}{l}\text { Tabla 2: Resultados obtenidos de las distintas } \\
\text { variables a estudio según la escala de Harris y } \\
\text { significación estadística de los mismos. } \mathrm{N}=78 \text {. }\end{array}$} \\
\hline Variable a estudio, (n) & $\begin{array}{l}\text { Puntuación media } \\
\text { escala de Harris }\end{array}$ & $\mathrm{p}$ \\
\hline \multicolumn{3}{|l|}{ Sexo } \\
\hline Mujer (48) & 79.3 & 0.081 \\
\hline Hombre (30) & 83.9 & \\
\hline \multicolumn{3}{|l|}{ Edad (años) } \\
\hline$<65(24)$ & 86.2 & 0.009 \\
\hline$\geq 65(54)$ & 79.1 & \\
\hline \multicolumn{3}{|l|}{$\mathrm{IMC}$} \\
\hline$<30(45)$ & 80.8 & 0.552 \\
\hline$\geq 30(33)$ & 82.2 & \\
\hline \multicolumn{3}{|l|}{ Cirugías previas } \\
\hline $1(47)$ & 83.4 & 0.031 \\
\hline$\geq 2(31)$ & 77.9 & \\
\hline \multicolumn{3}{|l|}{ Defecto óseo femoral } \\
\hline I, II, IIIA (61) & 82.6 & 0.005 \\
\hline IIIB (17) & 73.5 & \\
\hline \multicolumn{3}{|l|}{$\begin{array}{l}\text { Presencia de } \\
\text { stress-shielding }\end{array}$} \\
\hline Sí (28) & 81.2 & 0.922 \\
\hline No (50) & 81.5 & \\
\hline \multicolumn{3}{|l|}{ Complicaciones } \\
\hline Sí (19) & 76.3 & 0.024 \\
\hline No (59) & 83.0 & \\
\hline
\end{tabular}

visión acetabular con cotilo de doble movilidad); dos casos (2.6\%) de drenaje persistente de la herida quirúrgica solucionados mediante desbridamiento y antibioterapia empírica; y tres casos (3.8\%) de infección crónica que requirieron revisión en dos tiempos.

Los pacientes que sufrieron complicaciones intraoperatorias o postoperatorias alcanzaron un peor resultado funcional que quienes no registraron complicaciones $(\mathrm{p}=0.024)$ (Harris Hip Score de 76.3 y 83 puntos, respectivamente) (Tabla 2).

\section{Análisis de supervivencia}

Considerando como fracaso la revisión del vástago por aflojamiento aséptico, dos de los 78 casos (2.6\%) precisaron ser revisados durante el período de seguimiento obteniendo una supervivencia acumulada de 96.7\% (IC 95\%, de 92 a 100\%) a los 10 años (Figura 2A).

La tasa de supervivencia del implante femoral considerando como evento final la retirada del mismo por cualquier razón fue de 92\% (IC 95\%, 85.1 a 98.9\%) a los 10 años (Figura 2B).

La supervivencia con la revisión del vástago por cualquier motivo o migración del mismo superior a $5 \mathrm{~mm}$ como fracasos fue de $90.3 \%$ (IC 95\%, de 82.7 a $97.9 \%$ ) a los 10 años (Figura 2C). No objetivamos una disminución de la tasa de supervivencia desde los 116 meses postoperatorios hasta el final del seguimiento.

\section{Discusión}

La cirugía de revisión femoral constituye un procedimiento complejo en el que la elección del implante resulta fundamental. La combinación de un defecto óseo proximal, los cambios anatómicos en el fémur y el insuficiente stock óseo restante requiere de una estrategia de revisión dirigida a restaurar la reserva ósea o a baipasear el defecto obteniendo una fijación diafisaria distal.

Se han utilizado múltiples técnicas de reconstrucción femoral e implantes de revisión y no todos han tenido éxito. Estudios a largo plazo demuestran que la revisión con implantes cementados obtienen malos resultados, ${ }^{12,13,14}$ con elevadas tasas de aflojamiento y migración del vástago provocadas por una mala interdigitación mecánica del cemento en el hueso huésped. ${ }^{14,15}$ Los resultados obtenidos mediante el uso de vástagos no cementados con recubrimiento poroso proximal tampoco son esperanzadores, asociando tasas de revisión por aflojamiento aséptico superiores a $40 \%$ a corto plazo. ${ }^{16}$ Ello es debido a que el defecto óseo proximal presente en la mayoría de las cirugías de revisión impide la obtención de una fijación primaria estable así como de una correcta integración biológica a largo plazo. ${ }^{16,17,18}$

Para solventar este problema de integración se diseñaron los vástagos cilíndricos de recubrimiento completo de anclaje diafisario, con estructura tanto modular como monobloque, con buenos resultados presentados en la literatura 
en defectos femorales menores (I, II y IIIA de Paprosky). Estos vástagos de revisión permiten al cirujano «puentear» el defecto óseo femoral proximal obteniendo la fijación a nivel del istmo del fémur. Algunos diseños, como el utilizado en nuestra serie, incorporan un collarete proximal con el fin de incrementar la estabilidad axial y rotacional y mejorar la transmisión de fuerzas al calcar femoral, lo cual es de mayor importancia con este tipo de vástago respecto a los diseños cónicos. En nuestra serie observamos una tasa de osteointegración de 96.2\% de los casos. Otros autores revelan tasas de integración de entre 82 y 97\%.19,20,21 En estos estudios, de la misma manera que en este trabajo, se excluyen defectos
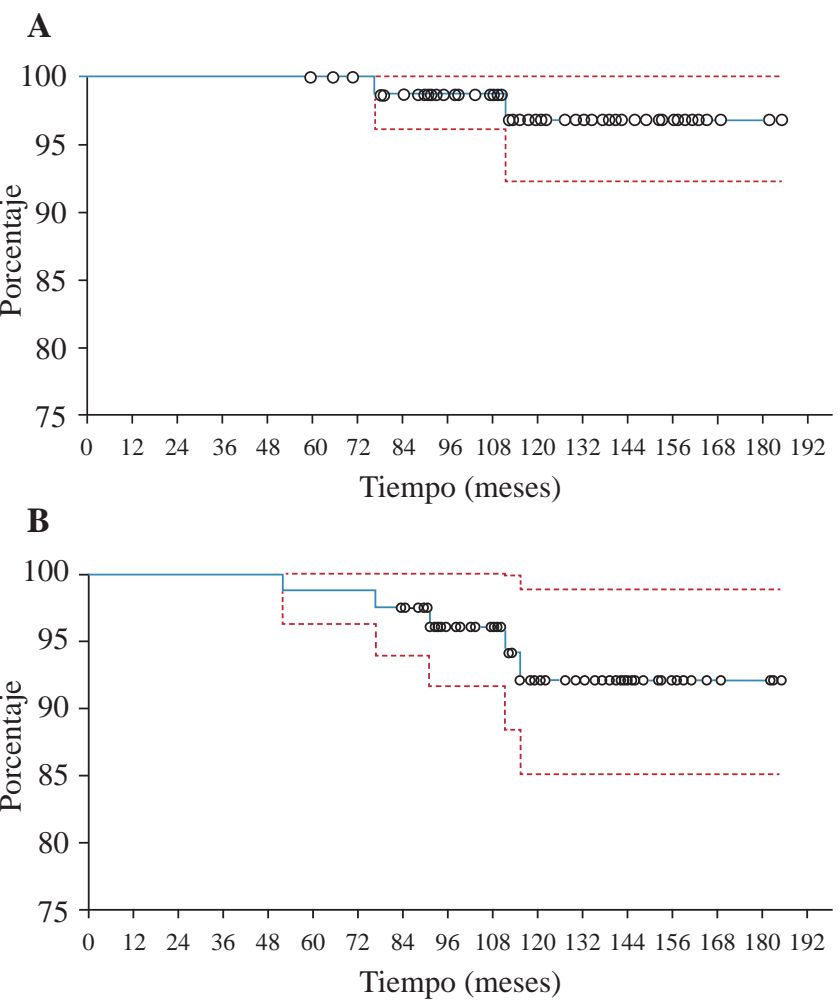

C

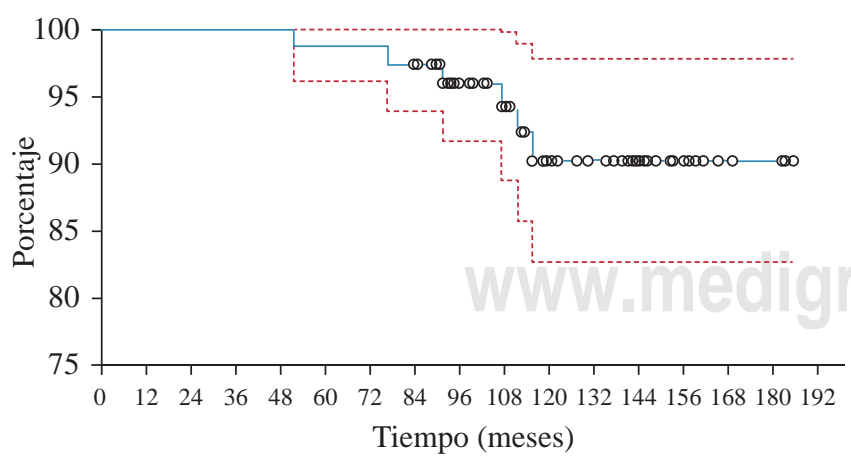

Figura 2: Curva de supervivencia acumulada de Kaplan Meier. A) Considerando como fracaso la retirada del vástago por aflojamiento aséptico, B) Considerando como fracaso la retirada del vástago por cualquier razón. C) Considerando como fracaso la revisión del vástago por cualquier motivo o migración del mismo superior a $5 \mathrm{~mm}$. óseos tipo IV de Paprosky. Weeden y Paprosky muestran una tasa de aflojamiento aséptico de $21 \%$ en pacientes con defectos óseos tipo IIIB. ${ }^{21}$ En nuestra serie un total de tres casos de los 45 (6.7\%) con defectos óseos tipo III presentaron un aflojamiento del vástago (dos aflojamientos en defectos tipo IIIB y un aflojamiento en un defecto tipo IIIA). Además, en nuestra serie obtenemos mejores resultados funcionales en aquellos pacientes con defectos óseos femorales menores respecto a los pacientes con defectos femorales mayores $(\mathrm{p}=0.005)$.

Sporer y Paprosky sugieren en su trabajo que los fracasos dados en pacientes con defectos óseos mayores son debidos a la dificultad de lograr una estabilidad o fijación primaria inicial. ${ }^{22}$ Además, concluyen que el uso de vástagos con un diámetro mayor de $18 \mathrm{~mm}$ es un factor de riesgo del fracaso de la osteointegración de los vástagos monobloque cilíndricos. De la misma manera, Garbuz sugiere que los vástagos cónicos acanalados son los indicados para aquellos casos en los que no es posible conseguir $4-5 \mathrm{~cm}$ de contacto diafisario o si el diámetro del vástago ha de ser mayor de $18 \mathrm{~mm} .^{23}$ Estos vástagos se caracterizan por una estructura cónica que favorece una fijación axial combinada con la estabilidad rotacional dada por la estructura acanalada. ${ }^{24,25,26}$ En nuestra serie el mayor diámetro utilizado fue de $17 \mathrm{~mm}$ en dos casos con un defecto óseo mayor en fémur proximal (Tabla 1).

En la última década ha aumentado el uso de los implantes modulares por su capacidad de conseguir fijación primaria proximal y distal de manera independiente así como una óptima versión y un correcto offset tanto vertical como lateral. ${ }^{24}$ Sin embargo, la modularidad no está exenta de riesgos, con fracasos de hasta $20 \%$, destacando la corrosión a nivel del sitio de unión de los componentes proximal y distal y la rotura. $^{23,27}$

Las fracturas intraoperatorias y las perforaciones del fémur son la complicación más frecuente en la cirugía de revisión femoral no cementada ${ }^{28}$ con tasas de 9 a $46 \%{ }^{29,30,31}$ Objetivamos dicha complicación en nueve casos (11.5\%), todas ellas dadas en defectos femorales tipo III. Debido a la alta tasa de fracturas femorales se refuerza la necesidad de una exposición femoral óptima y una inserción muy cuidadosa del vástago. Para minimizar esta complicación, Weeden y Paprosky recomiendan el uso de vástagos lo más cortos posibles y que por tanto eviten el arco femoral, siempre y cuando sea posible obtener una adecuada fijación primaria. ${ }^{21}$ En nuestra serie, en 20 de los 45 casos con defectos óseos tipo III se han utilizado los vástagos del mayor tamaño, estando disponibles los tamaños 155, 205 y 255 mm (Tabla 1). Es de suma importancia evitar complicaciones intraoperatorias o postoperatorias debido a los peores resultados funcionales obtenidos en los pacientes que presentan eventos adversos $(p=0.024)$. De la misma manera, obtuvimos peores resultados funcionales en los pacientes que habían sido intervenidos de la cadera revisada en más de una ocasión ( $\mathrm{p}=0.031)$.

Aunque se han reportado casos con rotura del vástago en pacientes con defectos óseos severos, no encontramos esta 
complicación en nuestra serie. Busch, ${ }^{32}$ en su serie de 219 revisiones con vástagos cilíndricos de recubrimiento completo, identifica cinco roturas de vástagos (2.3\%), las asocia a un defecto de soporte óseo proximal a un IMC $>30$ y al uso de una OTA. Las tensiones más altas dadas en el vástago son a nivel del sitio de la osteotomía, por lo que el uso de aloinjerto estructural reducirá la tensión en el vástago 48\%, recomendando esta técnica en pacientes con un defecto óseo severo asociado a una OTA. En nuestra serie realizamos 19 OTAs, siendo reparadas en todos los casos mediante cerclajes asociados en tres ocasiones a placa de grandes fragmentos y en 11 a aloinjerto estructural de banco (Figura 1).

El stress-shielding no ha constituido un problema significativo en nuestro estudio, estando presente en $35.9 \%$ de los casos. Las series disponibles en la bibliografía describen una tasa de dolor en el muslo de hasta $10 \% .{ }^{6}$ En nuestra serie siete pacientes $(9.3 \%)$ mostraron dolor a nivel del muslo, se presentó en dos de los tres vástagos aflojados (66\%) y en cinco de los vástagos osteointegrados (6.7\%); podemos equiparar estos resultados con los revelados por Moreland y Moreno ${ }^{33}$ con dolor en el muslo en $7 \%$ de los vástagos estables, en $16 \%$ con fijación fibrosa estable y en $75 \%$ de los aflojados. Esta complicación ha sido clásicamente atribuida al endocrecimento óseo, al diámetro y a la longitud del vástago. ${ }^{34}$

Obtuvimos una supervivencia acumulada de $96.7 \%$ a 10 años si consideramos como fracaso la retirada del vástago por aflojamiento aséptico del vástago y de $90.3 \%$ si consideramos fracaso la reintervención quirúrgica por cualquier otra causa o la migración del vástago mayor de $5 \mathrm{~mm}$. Esta tasa de supervivencia es comparable con la presentada por otros autores. $5,24,28,35$

En cuanto a las limitaciones de nuestro estudio, en primer lugar se trata de una serie de casos analizada de manera descriptiva y retrospectiva en la cual no hemos realizado un estudio comparativo con otra técnica quirúrgica, por lo cual está sujeto a las limitaciones que este tipo de estudio produce. Por otra parte, no controlamos las variables de interacción y confusión, por lo que las diferencias halladas en los resultados pueden deberse a causas distintas al tratamiento quirúrgico. Como ventajas de este estudio consideramos que el número de casos y su heterogeneidad son representativos para una unidad que atiende este tipo de cirugía de manera habitual.

\section{Conclusión}

Con los resultados obtenidos consideramos que los vástagos monobloque de recubrimiento completo constituyen una opción vigente y válida en cirugía de revisión de defectos femorales tipo I, II y IIIA de Paprosky proporcionando una supervivencia mayor de 90\% a largo plazo. En defectos más severos (tipo IIIB y IV), dados los peores resultados funcionales así como la mayor tasa de revisión descrita con los vástagos monobloque cilíndricos de recubrimiento completo, deberá considerarse el uso de otro tipo de implantes.

\section{Referencias}

1. Kurtz S, Ong K, Lau E, Mowat F, Halpern M. Projections of primary and revision hip and knee arthroplasty in the United States from 2005 to 2030. J Bone Joint Surg Am. 2007; 89: 780-5.

2. Ong KL, Mowat FS, Chan N, Lau E, Halpern MT, Kurtz SM. Economic burden of revision hip and knee arthroplasty in Medicare enrollees. Clin Orthop Relat Res. 2006; 446: 22-8.

3. Amanatullah DF, Howard JL, Siman H, Trousdale RT, Mabry TM, Berry DJ. Revision total hip arthroplasty in patients with extensive proximal femoral bone loss using a fluted, tapered, modular femoral component. Bone Joint J. 2015; 97: 312-7.

4. Sheth NP, Nelson CL, Paprosky WG. Femoral bone loss in revision total hip arthroplasty: evaluation and management. J Am Acad Orthop Surg. 2013; 21(10): 601-2.

5. Thomsen PB, Jensen NJF, Kampmann J, Hansen TB. Revision hip arthroplasty with an extensively porous-coated stem-excellent longterm results also in severe bone stock loss. Hip Int. 2013; 23: 353-8.

6. O’Shea K, Quinlan JF, Kutty S, Mulcahy D, Brady OH. The use of uncemented extensively porous-coated femoral components in the management of Vancouver $\mathrm{B}_{2}$ and $\mathrm{B}_{3}$ periprosthetic femoral fractures. J Bone Joint Surg Br. 2005; 87: 1617-21.

7. Harris WH. Traumatic arthritis of the hip after dislocation and acetabular fractures: treatment by mold arthroplasty. An end-result study using a new method of result evaluation. J Bone Joint Surg Am. 1969; 51(4): 737-55.

8. Engh CA, Massin P, Suthers KE. Roentgenographic assessment of the biologic fixation of porous-surfaced femoral components. Clin Orthop Relat Res. 1990; 257: 107-28.

9. Engh CA, Bobyn JD. The influence of stem size and extent of porous coating on femoral bone resorption after pri- mary cementless hip arthroplasty. Clin Orthop Relat Res. 1988; 231: 7-28.

10. Brooker AF, Bowerman JW, Robinson RA, Riley LH Jr. Ectopic ossification following total hip replacement. Incidence and a method of classification. J Bone Joint Surg Am. 1973; 55(8): 1629-32.

11. Brady OH, Garbuz DS, Masri BA, Duncan CP. Classification of the hip. Orthop Clin North Am. 1999; 30(2): 215-20.

12. Mulroy WF, Harris WH. Revision total hip arthroplasty with the use of so-called second-generation cementing techniques for aseptic loosening of the femoral component. A fifteen-year-average follow-up study. J Bone Joint Surg Am. 1996; 78(3): 325-30.

13. Kavanagh BF, Ilstrup DM, Fitzgerald RH. Revision total hip arthroplasty. J Bone Joint Surg Am. 1985; 67(4): 517-26.

14. Pellicci PM, Wilson PD Jr, Sledge CB, Salvati EA, Ranawat CS, Poss $\mathrm{R}$, et al. Long-term results of revision total hip replacement. $J$ Bone Joint Surg Am. 1985; 67: 513-6.

15. Franzen H, Toksvig-Larsen S, Lidgren L, Onnerfalt R. Early migration of femoral components revised with impacted cancellous allografts and cement. A preliminary report of five patients. J Bone Joint Surg Br. 1995; 77(6): 862-4.

16. Berry DJ, Harmsen WS, Ilstrup D, Lewallen DG, Cabanela ME. Survivorship of uncemented proximally porous-coated femoral components. Clin Orthop Relat Res. 1995; 319: 168-77.

17. Mulliken B, Rorabeck C, Bourne R. Uncemented revision total hip arthroplasty: a 4- to 6-year review. Clin Orthop Relat Res. 1995; 325: 156-62.

18. Malkani AL, Lewallen DG, Cabanela ME, Wallrichs SL. Femoral component revision using an uncemented, proximally coated, longstem prosthesis. J Arthroplasty. 1996; 11 (4): 411-8.

19. Della Valle CJ, Paprosky WG. Classification and an algorithmic approach to the reconstruction of femoral deficiency in revision total hip arthroplasty. J Bone Joint Surg Am. 2003; 85(Suppl 4): 1-6.

20. Krishnamurthy AB, MacDonald SJ, Paprosky WG. 5- to 13-year follow-up study on cementless femoral components in revision surgery. J Arthroplasty. 1997; 12(8): 839-47.

21. Weeden SH, Paprosky WG. Minimal 11-year follow-up of extensively porous-coated stems in femoral revision total hip arthroplasty. $J$ Arthroplasty. 2002; 17(Suppl 1): 134-7.

22. Sporer SM, Paprosky WG. Revision total hip arthroplasty: the limits of fully coated stems. Clin Orthop. 2003; 417: 203-9. 
23. Garbuz DS, Toms A, Masri BA, Duncan CP. Improved outcome in femoral revision arthroplasty with tapered fluted modular titanium stems. Clin Orthop Relat Res. 2006; 453: 199-202.

24. Van Houwelingen AP, Duncan CP, Masri BA, Greidanus NV, Garbuz DS. High survival of modular tapered stems for proximal femoral bone defects at 5 to 10 years followup. Clin Orthop Relat Res. 2013; 471(2): 454-62.

25. Fink B, Urbansky K, Schuster P. Mid term results with the curved modular tapered, fluted titanium Revitan stem in revision hip replacement. Bone Joint J. 2014; 96(7): 889-95.

26. Regis D, Sandri A, Bonetti I. Long-term results of femoral revision with the Wagner Self-Locking stem. Surg Technol Int. 2013; 23: 243-50.

27. Konan S, Garbuz DS, Masri BA, Duncan CP. Non-modular tapered fluted titanium stems in hip revision surgery. Bone Joint J. 2014; 96: 56-9.

28. Lachiewicz PF, Soileau ES. What is the survivorship of fully coated femoral components in revision hip arthroplasty? Clin Orthop Relat Res. 2015; 473: 549-54.

29. Chappell JD, Lachiewicz PF. Fracture of the femur in revision hip arthroplasty with a fully porous-coated component. J Arthroplasty. 2005; 20: 234-8.

30. Egan KJ, DiCesare PE. Intraoperative complications of revision hip arthroplasty using fully porous coated straight cobalt-chrome femoral stem. J Arthroplasty. 1995; 10: 45-50.
31. Paprosky WG, Greidanus NV, Antoniou J. Minimum 10-year-results of extensively porous-coated stems in revision hip arthroplasty. Clin Orthop Relat Res. 1999; 369: 230-42.

32. Busch CA, Charles MN, Haydon CM, Bourne RB, Rorabeck CH, Macdonald SJ, et al. Fractures of distally-fixed femoral stems after revision arthroplasty. J Bone Joint Surg Br. 2005; 87-B: 1333-6.

33. Moreland JR, Moreno MA. Cementless femoral revision arthroplasty of the hip: minimum 5 years followup. Clin Orthop Relat Res. 2001; 393: 194-201.

34. Ng FY, Chiu KY, Yau WP, Ng TP, Tang WM. Extensively coated femoral components in revision hip arthroplasty: followup study of 23 patients. J Orthop Surg. 2004; 12: 168-72.

35. Hamilton WG, Cashen DV, Ho H, Hopper RH, Engh CA. Extensively porous-coated stems for femoral revision. A choice for all seasons. $J$ Arthroplasty. 2007; 22(Suppl 1): 106-10.

Conflicto de intereses: No existe ningún conflicto de intereses relacionado con el artículo. 